\title{
Investigating Physician Acceptance of Telemedicine Technology: A Survey Study in Hong Kong
}

\author{
Paul Jen-Hwa Hu \\ Dept. of Information Systems \\ and Decision Sciences \\ University of South Florida
}

\author{
Olivia R. Liu Sheng \\ Dept. of Management \\ Information Systems \\ University of Arizona
}

\author{
Patrick Y. Chau, Kar-Yan Tam, \\ and Heidi Fung \\ Dept. of Information and \\ Systems Management, HKUST
}

\begin{abstract}
Fast-growing interest in telemedicine and increased investment in its enabling technology have made physician technology acceptance a growing concern for development and management of telemedicine. In this study, we used Theory of Planned Behavior to investigate technology acceptance by physicians who practiced in public tertiary hospitals in Hong Kong. Our data supported the investigated theory, whose explanatory power of physicians' technology acceptance was moderate, however. Overall, physicians showed positive attitudes towards use of telemedicine technology and exhibited moderate intention to use it, particularly for clinical tasks. Furthermore, several implications for development and management of telemedicine can also be drawn from the findings.
\end{abstract}

\section{Introduction}

Development of telemedicine in Hong Kong has accelerated in the past few years, as evidenced by a fastgrowing number of telemedicine programs that have been implemented for various purposes [1,2]. Currently, several institutions are delivering telemedicine-assisted healthcare services and many others are planning or initiating the technology implementation required for such services. Physicians have become increasingly aware of telemedicine and many of them have exhibited varying degrees of interest in using the technology in their practices. The recent inauguration of the Hong Kong Telemedicine Association (HKTA) demonstrates the level of collective interest in telemedicine within the Hong Kong medical community.

Physicians are arguably the most important users of telemedicine technology and their technology acceptance often has a crucial impact on a program's ultimate success. The telemedicine literature has attributed many program failures to lack of physician technology acceptance [3]. In most cases, a physician's decision to use or not to use a telemedicine technology available in his or her organization has been autonomous; it is likely to remain so in the future. At the dawn of large-scale telemedicine implementation in Hong Kong and elsewhere, it is essential to address the physician technology acceptance issue.

The predominant focus of most previous telemedicine research has been on technological developments or clinical applications; thus, offering limited discussion of managerial issues that include physician technology acceptance [4]. As Perenia and Allen commented [4], success of telemedicine requires an adopting organization to address both technological and managerial challenges. In response to this research void as well as practical needs, we used Theory of Planned Behavior (TPB) [5] to investigate acceptance of telemedicine technology by physicians in Hong Kong. Specifically, we examined the theory's overall explanatory power as well as its posited individual causal paths and considered their implications for development and management of telemedicine. Furthermore, we also assessed the current technology usage level among adopting physicians and compared their intention to use (i.e., continue using) the technology with that of potential adopting physicians.

\section{Literature Review and Research Motivation}

Essentially, telemedicine is about use of information and telecommunication technologies to support healthcare services, or related activities and tasks [6,7]. Pioneering telemedicine projects began nearly four decades ago by forward-looking physicians and researchers driven by requests for concept proving and feasibility assessment [8,9]. Most early telemedicine endeavors failed to meet expectations, as a result of several factors that included nascent and mostly primitive information infrastructure, unsophisticated technology, premature funding termination, and lack of user acceptance [3]. 
Recent technological advancements, together with continual increases in the performance-price index, have considerably increased the technical feasibility, clinical viability and financial affordability of telemedicine $[10,11]$. At the same time, persistent healthcare problems related to access, quality, and costs have also promoted telemedicine's becoming economically attractive, socially desirable and politically appealing $[3,12]$. As a result, a strong resurgence of interest in telemedicine has been under way around the globe [13-15].

Compared with implementations elsewhere, telemedicine development in Hong Kong has been unique in several ways [1]. First, all existing programs are implemented in densely populated areas where traffic congestion, not geographic dispersion, was a central concern for health service delivery. Second, all existing programs target tertiary care, a care sector where there exists considerable service needs. Third, most Hong Kong-based telemedicine programs are relatively small in scale, as measured by geographic coverage and resource requirements. In addition, government policy and regulation of telemedicine-assisted services are still emerging. These characteristics, combined with an acrossthe-board substantial government subsidy for healthcare services, have considerably reduced program implementation complexity and friction, making physician technology acceptance an increasingly critical factor for program success.

The potential impacts of telemedicine programs, existing or soon-to-be-implemented, and lessons learned from previous program failures have jointly demanded proper addressing of physician technology acceptance. Broadly speaking, development of telemedicine in Hong Kong is in an early adoption stage, as measured by limited but fast-growing aggregate adoption. As large-scale program implementation by healthcare organizations proliferates, it is essential to investigate physician technology acceptance, a paramount determinant of subsequent technology diffusion and ultimate program success.

Previous research has offered limited discussion of physician technology acceptance [4]. A handful of studies have investigated physician (user) technology acceptance issue, but most of them were limited in terms of medical specialty or sample size and were conducted in an ad hoc manner [16-20]. As summarized by Mitchell et al. [17], these investigations typically had a small and restrictive sample size and tested hypotheses that were idiosyncratically formulated without theoretical foundation.

Although user technology acceptance has received fairly extensive attention from information systems (IS) researchers and practitioners alike [21-23], what is needed is the bridging between investigation of physician technology acceptance and the existing IS literature of user technology acceptance. One dominant approach to user technology acceptance is intention-based, positing that an individual's technology acceptance is a conscientious act and therefore can be satisfactorily explained and predicted by his or her intention [24]. Results of our pre-study interviews showed that an intention-based approach appeared to encompass most of the technology acceptance decision factors identified by the physicians interviewed and therefore was chosen for this study over other approaches suggested by the literature [25].

\section{Target Physicians and Investigated Theory}

Target Physicians: This study was conducted using the survey methodology and was administered with physicians of pre-selected specialties at public tertiary hospitals in Hong Kong. Choice of physicians was made on the basis of the likelihood of their involving with telemedicine in the near future. As a group, these physicians have considerable interorganizational service needs which can be greatly assisted by telemedicine technology. Broadly speaking, Hong Kong has limited specialists and sub-specialists, making tertiary care a particular sector with considerable service queues or unmet service requests. Public tertiary hospitals appear more receptive to telemedicine than other healthcare organizations, public or private. These hospitals are pivotal in the Hong Kong healthcare system and usually have a fairly sophisticated technology base and reasonable access to financial resources. Individual physicians who practice at public tertiary hospitals tend to be innovative and often exhibit high interest in experimenting with new technologies and pioneering their use. Jointly, these factors make the target physicians more likely to become adopters (or early adopters) of telemedicine technology than their counterparts practicing at other healthcare organizations.

Several medical specialty areas were included in this study because of their frequent applications of telemedicine and accumulated satisfactory results [4]. Based on a continuum with high patient contact requirements on one end and intense medical image use on the other, the specialties included in the study were classified into three broad categories: highly patient-contact-based (e.g., Internal Medicine, Obstetrics and Gynecology, and Pediatrics), highly image-based (e.g., Radiology and Pathology), and mixed specialties which require a relatively balanced use of patient contacts and medical images (e.g., Accident and Emergency, and Surgery). Together, these specialties provided a desired diversity, which 
allowed us to investigate the potential impact of medical specialty on physician technology acceptance.

Theory of Planned Behavior: Ajzen [5] extended Theory of Reasoned Action (TRA) [26] to develop Theory of Planned Behavior (TPB) in order to account for situations where individuals lack complete control over a target behavior. Similarly to TRA, TPB asserts that an individual's behavior can be sufficiently explained and therefore predicted by his or her behavioral intention (BI) to perform the behavior [5]. However, TPB considerably differs from TRA in two areas. First, TPB includes perceived behavioral control (PBC), a construct that reflects the impact of perceived internal and external constraints on (or facilitators to) a particular behavior by specifying a direct causal link from $\mathrm{PBC}$ to behavioral intention (BI) as well as to actual behavior. According to TPB, an individual's behaviors are a direct function of his or her BI and PBC. Our pre-study interview results suggested that several constraints, including training, access and rewards, may affect an individual physician's acceptance of telemedicine technology. Second, TPB also differs from TRA in the conceptualization of BI. In addition to attitude and subjective norms $(\mathrm{SN})$ as suggested by TRA, TPB also includes PBC as a determinant of BI. That is, BI is jointly determined by attitude, SN and PBC.

Attitude refers to an individual's positive or negative evaluative affect about performing a particular behavior [26]. In our context, a physician's attitude toward accepting telemedicine technology is determined by combination of his or her salient beliefs about the consequences of using the technology and evaluations of those consequences. Subjective norms (SN) mean an individual's perception of relevant others' opinions on whether he or she should or should not perform a particular behavior [26]. However, physicians who share the same or similar normative beliefs may vary in the extent to which they want or are willing to comply with relevant others' opinions on use of telemedicine technology. Perceived behavioral control (PBC) is a construct unique to TPB and refers to an individual's perceptions of the presence or absence of requisite resources or opportunities necessary for performing a behavior [27]. For instance, the intention of an individual physician to use telemedicine technology can be affected by training and access and the physician may consider training more important to his or her technology use than access, or vice versa.

TPB is more specific than TRA because of its including PBC. Conceivably, TPB may provide a fuller explanation than TRA for physician technology acceptance. Use of TPB to explain or predict user technology acceptance has prevailed; some representative studies include Mathieson [28], who examined users' intention to use a spreadsheet software; Taylor and Todd
[24], who investigated user behaviors in using a computer resource center. Findings from the past studies were largely satisfactory and, collectively, they suggested that TPB was capable of providing sufficient explanation or satisfactory prediction for user technology acceptance, including actual technology use.

\section{Research Approach, Design, and Data Collection}

This section details our research approach, design, and data collection procedures.

\subsection{Research Approach}

In this study, technology acceptance means an individual's psychological state toward his or her voluntary use of a particular technology [29]. Technology acceptance has a profound influence on actual technology use and often can sufficiently explain or predict it [28]. Currently, telemedicine development in Hong Kong remains in an early stage and therefore widespread technology use does not exist. Notwithstanding, investigations of physician technology acceptance are particularly essential at a time when a fast-growing number of organizations are implementing telemedicine technology or considering its adoption in the near future. To cope with the constraint of limited actual technology use, we adopted "intention to use" to measure physician technology acceptance. Use of behavioral intention to explain or predict actual behavior is expedient because of its theoretical foundation and empirical support [30]. Furthermore, we also analyzed the current telemedicine technology usage level among adopting physicians and compared their intention to continue using the technology with potential adopting physicians' intention to use it.

We took a user-reporting, self-assessment approach to collecting data, which was appropriate for investigating physicians' intention to use telemedicine technology. Use of self-reported data has been examined and gained considerable support. For example, Melone [31] concluded that user-reported data are advantageous in situations where perceptual measures cope more effectively than objective measures. Rice [32] has employed user-reported data in his investigation of communication network usage.

\subsection{Research Design}

The primary research methodology for this study was self-administered survey. The following details 
our research design, including theory selection as well as instrument development, validation, and pre-testing.

Pre-study Interviews: To gain insight into the decision factors relevant to physician technology acceptance, we conducted a series of face-to-face semistructured, open-ended interviews with 12 physicians from different target specialties and hospitals. These interviews were recorded and later transcribed for analysis. Collectively, the physicians identified a considerable number of decision factors, which can be classified into several categories that included subjective evaluation, external pressure and opinions of relevant others, and individual competence. To a great extent, these decision factor categories are consistent with the constructs included in TPB, suggesting its appropriateness for our study.

Instrument Development: Use of TPB to investigate physician technology acceptance is advantageous because of its well-researched and validated measurement inventory [24]. Measurements obtained from the literature were formulated into question items, using a seven-point Likert scale with one being strongly agree and seven being strongly disagree. Six additional question items, categorical and quantitative, were also included to measure actual technology usage among adopting physicians.

The validity of an instrument may not be persistent across different technologies or user groups [33], necessitating re-evaluation of measurements obtained directly from the literature. Instrument validation was evaluated first. A review panel consisting of three physicians from different specialties evaluated the instrument at face value and the results were satisfactory, suggesting that the instrument included representative questions drawn from a universal pool appropriate for each respective construct [33].

Desired balance and randomness were applied to the questionnaire design. That is, the question items were randomly arranged and half of them were properly negated. A balanced negation can reduce the potential ceiling (floor) effect that induces monotonous responses to question items designed to measure a particular underlying concept. A random arrangement, on the other hand, induces the attention of respondents who, as a result, may become increasingly alert to manipulated question items. To anchor the responses properly [34], the questionnaire provided a working definition of telemedicine and included referential materials containing information on and common examples of telemedicine technology. The review panel that assisted with face-value instrument validation also examined the formatted survey instrument to ensure its layout and wording being appropriate for the healthcare context and communicative to the targeted physicians.
Evaluation of Content Validity: A preliminary test was conducted to evaluate the instrument's content validity to ensure that question items designed for a particular construct would draw from subject responses stemming from the same source [33]. As such, respondents would perceive question items developed for the same construct in a similar manner and respond to them based on their individual evaluation, judgment, and/or experience. Content validity was assessed through a card sorting method [35] that involved three physicians who did not participate in the instrument development. The question items included were printed on $8 \times 6$ index cards, which were shuffled randomly and presented to the physicians, each of whom was asked individually to sort them into appropriate categories. Results from the card sorting were satisfactory and the physicians were able to categorize the question items correctly with an accuracy rate of 90 percent or better.

A Pre-test Study: With a satisfactory face-value instrument validity and content validity established, evaluation of construct validity and reliability proceeded [33]. Construct validity is an operational issue and concerns whether the measurements chosen are truly constructs that describe the phenomenon under investigation. On the other hand, reliability is an index to measurement accuracy and refers to the extent to which a respondent can answer the same or approximately the same questions the same way each time. A pre-test that included thirty-five physicians from different specialties and hospitals was performed. Physicians took part in the pre-test study were excluded from the subsequent formal study. Results of the pre-test study were fairly satisfactory, as manifested by reasonably high Cronbach's alpha values and relatively higher co-relations among measurements for the same construct than for a different construct. These results suggested that the instrument was of acceptable measurement reliability, and convergent and discriminant validity.

\subsection{Data Collection Procedures}

A total of 70 clinical departments at the target hospitals were contacted through an encounter letter that briefly described the intended study. Personal visits and phone calls were later made to the departments' chiefs of service to provide them with detailed study information and solicit their voluntary participation. Additional contacts and visits were made to those who showed reluctance at initial contact, some of whom eventually agreed to participate in the study. The participation rate of the study was 59 percent, including 41 of the 70 departments contacted. 
Questionnaire packets were delivered to the targeted physicians who were given at least two weeks to complete the questionnaire. A letter soliciting internal promotion of the study was faxed to chiefs of service of the participating departments immediately after questionnaire distribution. Physicians returned the completed questionnaires to their department secretaries, from whom the data were collected at a later time. Reminders and additional questionnaires were sent to physicians who had failed to return a completed questionnaire before the specified deadline. Late respondents were given another week to complete the questionnaires. A second reminder and another questionnaire were sent to the physicians who still had not responded within the extended response period. Finally, a terminating response window was indicated in a faxed reminder to the remaining nonrespondents, who were asked to mail in their completed questionnaires.

\section{Data Analysis and Results}

Of the 1,728 questionnaires distributed, 421 were completed and returned, showing a 24.4 percent response rate. As a group, the respondents averaged 34.7 years in age and had 9.4 years of post-internship clinical experience in their respective specialty areas. Among the respondents, the male-to-female ratio was approximately 4 to 1 , with 80 percent having received medical education in Hong Kong. Distribution of the respondents was fairly balanced among the participating hospitals, ranging from 6.5 to 11.6 percent. Similarly, distribution among the investigated specialties was fairly balanced as well, with the exception of radiologists and neurosurgeons who exhibited relatively high interest in the study.

\subsection{Evaluation of Non-response Biases}

Non-response is a potential source of bias in survey studies and therefore needs to be properly addressed [36]. Each distributed questionnaire had an internal code, which enabled us to identify non-respondents. However, access to information about non-respondents was denied by most participating departments. A commonly used alternative approach to assessing potential non-response biases is to compare early and late respondents along some important demographic dimensions. In this study, we compared early and late respondents using age, postinternship clinical experience, medical specialty, gender, and country where medical school was attended.

Among the responses returned, 49.4 percent were received within the regular response window. The early respondents and late respondents did not differ significantly in age (35.8 versus 33.5 years), postinternship clinical experience (10.7 versus 8.1 years), medical specialty, gender, or country where they attended medical school. Furthermore, these two groups were also comparable in the distribution of the respective home hospital.

\subsection{Analysis of Intention to Use Telemedicine Technology}

The level of intention to use telemedicine technology among the respondents was moderate, as summarized in Table 1. Most of the respondents indicated that they would use the technology when it became available in their departments but showed varying degrees of reluctance to use it routinely to provide healthcare services. In addition, the respondents also indicated that they would use telemedicine technology for different tasks, clinical or non-clinical.

An analysis of respondents' intention to use telemedicine technology showed no significant differences among the specialty categories investigated, as summarized in Table 1. Physicians whose specialties involved relatively balanced proportions of patient contacts and medical images exhibited a slightly stronger intention than physicians who needed more patient contacts or use of medical images in their practices. Further analysis showed that neurosurgeons were particularly enthusiastic about telemedicine technology. Consistent with the literature [4], our results also suggested that radiologists and pathologists appeared more receptive to telemedicine technology than physicians of patient-contact-based specialty. However, the difference was statistically insignificant. 


\begin{tabular}{|c|c|c|c|c|c|c|c|c|}
\hline \multirow[t]{2}{*}{ Question Item } & \multicolumn{2}{|c|}{ Overall } & \multicolumn{2}{|c|}{ Contact-based } & \multicolumn{2}{|c|}{ Image-based } & \multicolumn{2}{|c|}{ Mixed } \\
\hline & Mean & Std. & Mean & Std. & Mean & Std. & Mean & Std. \\
\hline Would use (A) when it becomes available in my department & 2.7 & 1.1 & 2.8 & 1.1 & 2.7 & 1.1 & 2.6 & 1.0 \\
\hline Would use (A) to provide healthcare services as often as needed & 3.5 & 1.3 & 3.5 & 1.3 & 3.5 & 1.2 & 3.4 & 1.4 \\
\hline Would use (A) for patient care and management routinely & 3.2 & 1.4 & 3.4 & 1.5 & 3.2 & 1.3 & 3.1 & 1.5 \\
\hline Would use (A) for patient care and management whenever possible & 3.0 & 1.3 & 3.1 & 1.3 & 3.0 & 1.1 & 2.9 & 1.4 \\
\hline Would use (A) to do different tasks, clinical or non-clinical & 3.1 & 1.1 & 3.2 & 1.1 & 3.0 & 1.1 & 3.0 & 1.1 \\
\hline Would use (A) for patient care and management frequently & 3.2 & 1.3 & 3.4 & 1.4 & 3.4 & 1.2 & 2.8 & 1.1 \\
\hline
\end{tabular}

\section{Table 1: Intention to use telemedicine technology - all responses and by specialty category}

Among the respondents, male physicians seemed to exhibit a slightly higher level of intention to use telemedicine technology than their female counterparts. However, the difference was insignificant statistically. Variations between early and late respondents in respective intention to use telemedicine technology were also examined. The results showed no significant differences, suggesting there was the desired comparability among the target physicians which, in turn, may reduce the threat of potential non-response biases.

Relationships of intention to use telemedicine technology with age as well as with post-internship clinical experience were also examined. An aggregate intention was obtained for each individual respondent by averaging his or her responses to all intention-oriented question items, with appropriate adjustments for those that were negated. The results showed a considerable positive correlation between intention and age. This might suggest that the older the physician the stronger his or her intention to use telemedicine technology. However, caution needs to be taken when interpreting this observed correlation because the respondents included in this study were relatively young, averaging 34.7 years in age. On the other hand, correlation between clinical experience and the investigated intention was not noticeable.

\subsection{Analysis of Actual Use of Telemedicine Technology}

About 14.7 percent (60 out of 407) of the respondents qualified as adopters by indicating previous or current use of telemedicine technology. However, their actual technology usage levels were mostly moderate and appeared to have a clinical focus, as shown in Table 2. On average, the adopters used telemedicine technology 6.2 times a week and telemedicine-assisted services accounted for only 7 percent of their total patient care. However, the intensity of technology usage was considerable. In a typical telemedicine-enabled service, use of the technology accounted for approximately 40 percent of the service provided.

\begin{tabular}{||l|c|c||}
\hline \multicolumn{1}{|c|}{ Question Item } & Mean & Std. \\
\hline Use (A) for patient care and management even when alternatives are available & 2.7 & 1.2 \\
\hline Use (A) for different clinical tasks or activities & 2.8 & 1.1 \\
\hline Use (A) for tasks or activities not directly related to patient care and management & 4.1 & 1.9 \\
\hline Use (A) --- times a week & 6.2 & 9.0 \\
\hline Use of (A) accounts for ---\% of my total patient care & $7.0 \%$ & $8.4 \%$ \\
\hline (A) accounts for ---\% of the provided service that involves (A) & $40.4 \%$ & $30.3 \%$ \\
\hline
\end{tabular}

Table 2: Summary of actual telemedicine technology usage

A review of the self-identified adopters showed that radiologists and surgeons, especially neurosurgeons, were the two dominant physician groups in pioneering use of telemedicine technology in Hong Kong. Jointly, they represented 53.4 percent of the adopters. This relatively high technology usage level is particularly significant when the number of radiologists and neurosurgeons practicing in Hong Kong is taking into account.

A comparative analysis of adopters and potential adopters showed that physicians who had used or were using telemedicine technology had considerably stronger intention to continue using it. Compared with potential adopters, the adopters showed greater interest in using the technology on a frequent and routine basis for both patient care and non-clinical tasks. This observed stronger intention level among adopting physicians reinforces TPB as well as other intention-based theories, which state that an individual's actual behavior can be explained by his or her intention for the behavior. The adopters and the potential adopters were similar in age and clinical experience and had a comparable distribution in gender, medical specialty 
and country where they attended medical school.

\subsection{Results of Model Testing}

Support of TPB for physician acceptance of telemedicine technology was tested using LISREL 8 with maximum likelihood estimation, which is appropriate for testing models that have a well-developed underlying theoretical foundation [37]. The overall model fit was evaluated using common fit indexes [38]. Predictive power of the model was examined using the resulting $\mathrm{R}^{2}$ for the dependent construct (i.e., intention to use telemedicine technology). Furthermore, the individual causal paths specified by TPB were also assessed in terms of observing coefficients and statistical significance.

\begin{tabular}{||l|c||}
\hline \multicolumn{1}{|c|}{ Measurement } & TPB \\
\hline Indexes to Overall Model Fit & \\
$\chi 2$ / degree of freedom & 1.74 \\
G.F.I. & 0.96 \\
A.G.F.I & 0.94 \\
N.N.F.I. & 0.95 \\
C.F.I. & 0.96 \\
R.M.S.E.A & 0.04 \\
\hline Explanatory Power of the model & \\
$\mathrm{R}^{2}$ BI & 0.37 \\
\hline
\end{tabular}

Table 3: Overall fit and explanatory power

Overall, our data supported TPB, as summarized in Table 3. The fit indexes suggested that the model provided a reasonably good fit to the collected responses. However, overall explanatory power of the model was moderate. Together, Attitude, SN, and PBC accounted for 37 percent of the variances in intention. As shown in Figure 1, most of the hypothesized causal paths were supported with statistical significance. A physician's attitude showed a significant positive effect on his or her intention to use the technology. Likewise, PBS had a considerable effect on intention as well. However, the hypothesized link from SN to intention was not supported and showed a negative effect which was contrary to what TPB suggests.

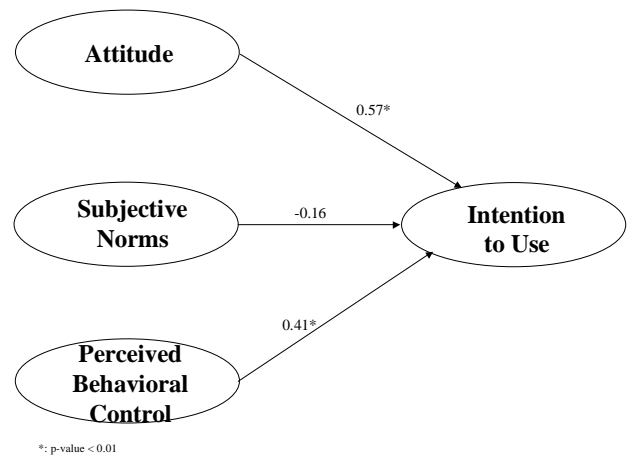

Figure 1: Theory testing results - TPB

\subsection{Implications for Telemedicine Development and Management}

Valuable implications for both telemedicine development and technology management can be drawn from this study. On the telemedicine development front, several interesting observations can be readily made from this study and their implications for telemedicine development are discussed as follows. First, neurosurgeons' surprisingly strong intention to use telemedicine technology and considerable actual technology usage level can be explained in part by their involvement with telemedicine development in Hong Kong. This implies that the medical community and professional associations can serve an important role in fostering telemedicine development by promoting, educating and involving member physicians with telemedicine technology and its usefulness, including clinical efficacy.

Critical mass is also essential for telemedicine development. This study showed a remarkable intention differential between radiologists and pathologists, which can be partially attributed to existence of a critical mass. Most telemedicine programs in Hong Kong include teleradiology; thus creating a critical mass in the radiology community. One effective way to create the critical mass needed to disseminate telemedicine technology among physicians is through their professional societies which play an instrumental role and can be leveraged for nourishing and cultivating a momentum to proliferate telemedicine technology among their members.

The adopting physicians seemed to have a clinical focus in their actual technology utilization, which may be explained in part by service load and clinical orientation commonly found in existing Hong Kongbased telemedicine programs. However, telemedicine should not and cannot be confined to clinical purposes. One interesting example is an Intranet-based radiology 
teaching theater that involves radiology departments at several hospitals in Hong Kong. Impacts of such nonclinical programs can be significant enough to broaden the frontier of telemedicine applications.

At the same time, several implications for technology management can also be drawn from the model testing results. First, attitude appeared to be an important determinant of physician technology acceptance and can be managed with peer-to-peer communication of the values and utilities of telemedicine. Information source and creditability are two essentials in such communications. Perceived behavioral control was also important in shaping physicians' technology acceptance and can be greatly facilitated by proper training, convenient access, and in-house technology expertise. Interestingly, subjective norm did not appear to have a significant impact on physician technology acceptance, which may be partially explained by professional dominance and practice autonomy. However, subjective norm may become increasingly relevant in a highly managed care system where third-party payers and patients have stronger voices in the physician's practice.

Furthermore, adopting physicians showed a stronger intention to use telemedicine technology than potential adopters, a difference that may have resulted from telemedicine's usefulness in supporting physicians' tasks and services. This may imply that management needs to consider making arrangements for member physicians to use the technology on a trial basis, or at least to interact with clinicians who use the technology routinely before committing resources to adoption of a particular telemedicine technology.

\section{Conclusion}

This study has contributed to development and management of telemedicine by investigating physician technology acceptance using a well-researched theory. On the research front, we have contributed to extending the validation of Theory of Planned Behavior, a salient intention-based theory for user technology acceptance. Our findings suggested the potential limitation of the theory's explanatory power of technology acceptance by physicians. Furthermore, this study also responded to the call for more research efforts in health care, a service sector that exhibits rapidly expanding IT investment and deployment.

To a great extent, our data supported TPB. However, caution needs to be taken when extrapolating the results. Responses to this study were voluntary and therefore inevitably subject to self-selection biases. Conceivably, physicians who were interested in, had used, or were currently using telemedicine technology may have been more likely to respond. The relatively high response rate from radiologists and neurosurgeons may show signs of the presence of self-selection. The sampling window also needs to be considered when interpreting the findings. Physicians who practiced at public tertiary hospitals in Hong Kong represented a very specific population, whose members may differ considerably from their counterparts practicing at front-line hospitals or clinics. Cultural differences can be another limitation. Conceivably, physicians may vary considerably from culture to culture in attitude formation and technology assessment. For instance, physicians from a culture characterized by relatively high uncertainty avoidance may perceive usefulness and user friendliness of telemedicine technology differently from those whose culture is more tolerant of uncertainty. These limitations, in turn, represent some of the directions for our future research.

\section{References:}

[1] Liu Sheng, O.R., Hu, P.J., Au, G., Higa, K, and Wei, C, "Urban Teleradiology in Hong Kong," Journal of Telemedicine and Telecare, Vol.3, No.2, 1997, pp.7177.

[2] Liu Sheng, O.R., Hu, P.J., Wei, C., Higa, K, and Au, G., "Adoption and Diffusion of Telemedicine Technology in Healthcare Organizations: A Comparative Case Study in Hong Kong," Journal of Organizational Computing and Electronic Commerce (forthcoming).

[3] Bashshur, R.L., Sanders, J., and Shannon G.W. (Ed.), Telemedicine: Theory and Practice, Charles Thomas, Springfield, IL, 1997.

[4] Perednia, D.A. and Allen, A., "Telemedicine technology and Clinical Applications," Journal of American Medical Association, Vol.273, No.6, 1995, pp.483-488.

[5] Ajzen, I., From Intentions to Actions: A Theory of Planned Behavior in Action Control: From Cognition to Behavior, edited by J. Kuhl and J. Beckmann, Springer Verlag, NY, 1985.

[6] Bashshur, R.L., "On the Definition and Evaluation of Telemedicine," Telemedicine Journal, Vol.2, No.1, 1995, pp.19-30.

[7] Field, M.J. (Ed.), Telemedicine: A Guide to Assessing Telemedicine in Health Care, National Academy Press, Washington, DC, 1996. 
[8] Jutra, A., "Teleroentgen Diagnosis by Means of Videotape Recording," American Journal of Roentgenology, Vol.82, 1959, pp.1099-1102.

[9] Wittson, C.L., Afflect, D.C., and Johnson, V., "Two-way Television Group Therapy," Ment Hosp., Vol.12, 1961, pp.22-23.

[10] Office of Rural Health Policy, Exploratory Evaluation of Rural Applications of Telemedicine, February 1997.

[11] U.S. Department of Commerce, Telemedicine Report to Congress, January 1997.

[12] Office of Technology Assessment, U.S. Congress, Bringing Health Care Online: The Role of Information Technologies, U.S. Government Printing Office, OTA-ITC-624, September 1995.

[13] International telecommunications Union, Telemedicine and Developing Countries, Document 2/155-E, Study Group, International Telecommunications Union, September 1996.

[14] Wright, D. and Androuchko, L., "Telemedicine and Developing Countries, " Journal of Telemedicine and Telecare, Vol.2, No.2, 1996, pp.63-70.

[15] Wootton, R., "The Possible Use of Telemedicine in Developing Countries," Journal of Telemedicine and Telecare, Vol.3, No.1, 1997, pp.23-26.

[16] Allen, A., Hayes, J., Sadasivan, R., Williamson, S.K., and Wittman, C., "A Pilot Study of the Physician Acceptance of Tele-oncology," Journal of Telemedicine and Telecare, Vol.1, No.1, 1995, pp.34-37.

[17] Mitchell, B.R., Mitchell, J.G., and Disney, A.P., "User Adoption Issues in Renal Telemedicine," Journal of Telemedicine and Telecare, Vol.2, No.2, 1996, pp.81-86.

[18] Mairinger, T., Gabl, C., Derwan, P., Mikuz, G., and FerrerRoca, O., "What Do Physicians Think of Telemedicine? A Survey in Different European Regions," Journal of Telemedicine and Telecare, Vol.2, No.1, 1996, pp.50-56.

[19] Gschwendtner, A., Netzer, T., Mairinger, B., and Mairinger, T., "What Do Students Think About Telemedicine?" Journal of Telemedicine and Telecare, Vol.3, No.3, 1997, pp.169-171.

[20] Mairinger, T., Netzer, T., Schoner, W., and Gschwendtner, A., "Pathologists' Attitudes to Implementing Telepathology," Journal of Telemedicine and Telecare, Vol.4, No.1, 1998, pp.41-46.

[21] Alavi, M. and Carlson, P., "A Review of MIS Research and Disciplinary Development," Journal of Management Information Systems, Vol.8, No.4, 1992, pp.45-62.

[22] Swanson, E.B. and Ramillar, N.C., "Information Systems Research Thematics: Submissions to a New Journal, 1987-
1992," Information Systems Research, Vol.4, No.4, 1993, pp.299-330.

[23] Niederman, J.F., Brancheau, J.C., and Wethebe, J.C., "Information Systems Issues for the 1990s," MIS Quarterly, 1991, pp.475-500.

[24] Taylor, S. and Todd, P.A., "Understanding Information Technology Usage: A Test of Competing Models," Information Systems Research, Vol.6, No.1, 1995, pp.144-176.

[25] Rogers, E.M., The Diffusion of Innovation, 4th edition, Free Press, New York, NY, 1995.

[26] Fishbein, M. and Ajzen, I., Belief, Attitude, Intention and behavior: An Introduction to Theory and Research, Addison-Wesley, Reading, MA, 1975.

[27] Ajzen, I. and Madden, T.J., "Prediction of Goaloriented Behavior: Attitude, Intention, and Perceived Behavioral Control," Journal of Experimental Social Psychology, Vol.22, 1986, pp.453-474.

[28] Mathieon, K., "Predicting User Intention: Comparing the Technology Acceptance Model with Theory of Planned Behavior," Information Systems Research, Vol.2, No.3, 1991, pp.173-191.

[29] Gattiker, U.E., Managing Computer-based Office Information Technology: A Process Model for Management in Human Factors in Organizational Design, edited by H. Hendrick and O. Brown, NorthHolland, Amsterdam, The Netherlands, 1984.

[30] Sheppard, B.H., Harwick, J., and Warshaw, P.R., "The Theory of Reasoned Action: A Meta-analysis of Past Research with Recommendation for Modification and Future Research," Journal of Consumer Research, Vol.15, 1988, pp.325-343.

[31] Melone, N.P., "Theoretical Assessment of Usersatisfaction Construct in Information Systems Research," Management Science, Vol.36, 1990, pp.7691.

[32] Rice, R.E., "Computer-mediated Communication System Network Data: Theoretical Concerns and Empirical Examples," International Journal of Manmachine Studies, Vol.32, 1990, pp.627-647.

[33] Straub, D.W., "Validating Instruments in MIS Research,” MIS Quarterly, Vol.13, 1989, pp.147-169.

[34] Hufnagel, E.M. and Conca, C., "User Response Data: The Potential for Errors and Biases," Information Systems Research, Vol.5, No1, 1994, pp.48-73.

[35] Moore, G.C. and Benbasat, I., "Development of an Instrument to Measure the Perception of Adopting an 
Information Technology Innovation," Information Systems

Research, Vol.2, No.3, 1991, pp.192-223.

[36] Fowler, F.J., Survey Research Methods, 2nd edition, SAGE, Thousand Oaks, CA, 1993.

[37] Joreskog, K.G., Testing Structural Equation Models in Testing Structural Equation Models, edited by K. Bollen and S. Long, SAGE, Thousand Oaks, CA, 1993.

[38] Hu, L. and Bentler, P.M., Evaluating Model Fit in Structural Equation Modeling: Concepts, Issues, and Applications, edited by R.H. Hoyle, SAGE, Thousand Oaks, CA 1995. 